\title{
Tao-Hong-Si-Wu Decoction improves depressive symptoms in model rats via restorations of neural transmitters and ameliorations of BDNF-CREB- arginase I axis disorders
}

\author{
Xiaoping Zhang \\ Anhui Medical University \\ Zeng Li \\ Anhui Medical University \\ Chuanpu Shen \\ Anhui Medical University \\ Jinzhi He \\ Anhui Medical University \\ Longfei Wang \\ Anhui Medical University
}

Lei Di

Anhui Medical University

Bin Rui

Henry Ford Medical Group: Henry Ford Health System

Ning Li

Anhui Medical University

Liu Zhicheng ( $\square$ liuzhicheng@ahmu.edu.cn )

Anhui Medical University https://orcid.org/0000-0001-6539-511X

\section{Research}

Keywords: depressive disorder, metabolomics, GC-MS, traditional Chinese Medicine

Posted Date: October 12th, 2021

DOI: https://doi.org/10.21203/rs.3.rs-915908/v1

License: () (1) This work is licensed under a Creative Commons Attribution 4.0 International License. Read Full License 


\section{Abstract \\ Background}

Depressive disorder (DD) has become a global health problem. Applications of Chinese medicine have been demonstrated to be potential in the treatment of DD. Tao-Hong-Si-Wu decoction (TSD), a traditional Chinese medicine formula is widely used to treat ameliorate anemia, liver and heart dysfunctions, and inflammation. Such symptoms are also associated with DD. Hence, our initial hypothesis was to observe and explore the targets of the effect of TSD on DD.

\section{Methods}

The DD model was established by chronic unpredictable mild stress (CUMS) in rats. The measurements of body weight and behavioral tests were performed to confirm the success of modeling and observe the effect of TSD on the model animals. A gas-chromatography coupled with mass spectrometry (GC-MS)based metabolomic analysis was conducted to reveal the metabolic characteristics related to the curative effect of TSD. Serum serotonin and arginase I (Arg I), which are associated with the key feature metabolites responsible for the effect of TSD on depression, were assessed by an ELISA assay. Proteins in the Brain-derived neurotrophic factor (BDNF)/ Tropomyosin receptor kinase B (TrkB)/cAMP response element-binding protein (CREB) signaling, which lead to the regulations of Arg I, were analyzed using Western blot assay.

\section{Results}

Body weight losses and increased immobility durations in the behavioral tests were observed in the CUMS rats while such features were attenuated in the TSD-treated rats. General decreases in serum amino acids and energetic metabolites were found in CUMS rats. Significant increased ornithine and urea, the products of arginase degradation, were also characterized in the DD-mimicked rats. These metabolic dysregulations were reversed in the TSD-treated groups. Upregulated Arg I and downregulated serotonin were observed in the model group but the dysregulations were improved in the TSD-treated groups. Compared with the blank control, lower expressions of BDNF, TrkB, extracellular signal-regulated kinases (ERK), p-ERK, and CREB were found in the hippocampus of CUMS rats. Expressions of the proteins were restored in all the TSD-treated groups.

\section{Conclusions}

TSD improves depression-like symptoms in CUMS rats, which may be primarily related to its effect both on enhancing the release of neural transmitters such as glycine and serotonin and on the improvement of disorders of the BNDF-CREB-Arg I axis. 


\section{Introduction}

Depressive disorder (DD) is a common illness that severely limits psychosocial function and increases the risk of suicide [1]. According to a report by World Health Organization in 2017, there are about 322 million patients with depression worldwide. The high prevalence and risk of disability make the disease be a serious global health problem [2].

The pharmacotherapies for DD are primarily based on the enhancement of monoamine neurotransmission until the present. However, few drugs combine effectiveness and tolerability [3]. This is partly due to the unclear pathology of DD for the moment. Typically, more than the reductions of monoamine neurotransmitters, physiological disorders can be also found in other pathways in DD such as dysfunctions of the hypothalamic-pituitary-adrenal (HPA) axis, inflammation, inhibition of neurogenesis, loss of brain function, and genetic modifications. Thus, multi-target medications may be a promising therapeutic strategy for DD treatment.

Emerging evidence shows that ethnomedicines, which are generally multi-targeted, especially Chinese traditional medicine (CTM), play roles as important complementary therapies in DD treatment [4]. Several inherited prescriptions from TCM have been reported to be useful to improve depressive symptoms [5-8]. It is worth noting that most herbal drugs show a common effect of serotonin enrichment, which is likely to be a common effect of most TCM prescriptions on DD [9]. According to the theory of TCM, DD is associated with the deficient circulation of blood and qi, invasion of detrimental qi, and organ weaknesses, which can be implicated with the depressive symptoms of anemia and malnutrition, inflammation, and impaired organ functions, respectively [10-12]. Interestingly, these conditions were reported to be improved by Tao-Hong-Si-Wu decoction (TSD), a classical formula recorded in TCM, which consists of Semen Prunus (Amygdalus persica L.), Flos Carthami (Carthamus tinctorius L.), Rhizoma Chuanxiong (Ligusticum chuanxiong Hort.), Radix Angelicae sinensis (Angelica sinensis (Oliv.) Diels), Radix Rehmanniae praeparata (Rehmannia glutinosa (Gaert.) Libosch. ex Fisch. et Mey.), and Radix Paeoniae Alba (Paeonia lactiflora Pall.). The traditional use of TSD was to treat ischemic stroke [13]. In the past decades, bioactive compounds contained in these herbs such as ferulic acid, albiflorin whose effects have been revealed in improving inflammation and depressive symptoms [14, 15]. However, no previous studies focused on the antidepressant function of TSD. In the current study, our initial hypothesis was to investigate whether TSD is useful to improve DD.

It is challenging to clarify the mechanism behind the curative effect of a TCM formula because of the comprehensive ingredients contained in the mixture of various herbs. To this end, metabolomics is applied as a promising tool since the improvement of the disease-induced dysregulations can be witnessed sensitively and simultaneously by the restorations of metabolic disorders [16]. Also, variations in inherent metabolites can appear individual responses to treatments thereby providing evidence for personalized therapy. In addition, an overview of the regulations in the metabolome is following the theory of TCM which emphasizes restoring the integral balance and harmony for the internal environment of patients [17]. However, the metabolic profiling is prone to be varied by environmental variations and 
individual differences. Further verifications in the upstream proteins related to the drug effect-induced metabolic variations are necessary to ascertain the medicinal actions and corresponding drug targets [18].

Herein, we first confirmed the antidepressant effect of TSD on the DD animal model by bodyweight measurements and behavioral tests. A series of serum metabolomic analyses were conducted subsequently to reveal discriminant metabolites and varied metabolic pathways associated with the CUMS modeling and the effect of TSD on DD. Feature metabolites-associated upstream proteins in serum and hippocampus were tested to explore the mechanism behind TSD effects on DD.

\section{Materials And Methods \\ 2.1. Experimental Animals}

Forty-eight male SD rats weighing 200-250g were purchased from the Experimental Animal Center of Anhui Medical University (Hefei, China). Animals were housed while free access to food and water was allowed for 1 week.

\subsection{Medication}

All the herbs were purchased from Anhui Baixingyuan Pharmacy, Hefei, China. The herbs were mixed before decoction with the mass proportion of each herbal medicine as follows: Semen Prunus 12g, Flos Carthami 18g, Rhizoma Chuanxiong 18g, Radix Angelicae sinensis 18g, Radix Rehmanniae praeparata $18 \mathrm{~g}$, and Radix Paeoniae Alba 18g. according to the original record of "Yuji Weiyi", a Chinese classical medical book.

\subsection{Chemicals}

HPLC grade methanol, isopropanol, acetonitrile, methoxamine salt, pyridine, pentobarbital, and N-paraffin mix that was used for calculating retention index were purchased from J\&K Scientific Ltd. (Shanghai, China); HPLC grade N-methyl-N-trimethylsilyltrifluoroacetamide (MSTFA), and the standard substances including L-norvaline, L-lysine, L-tyrosine, L-phenylalanine, L-serine, L-valine, L-glutamic acid, L-alanine, Lproline, L-ornithine, D-glucose, sucrose, urea, and lactic acid, and fluoxetine were purchased from SigmaAldrich Ltd. (Shanghai, China). The standard products of the six herbs included in TSD were bought from Desite Company (Chengdu, China). Ultrapure water was prepared by the Milli-Q system (Merck, Darmstadt, Germany).

\subsection{Preparation and the evaluation of the homogeneity of TSD}

Herbs contained in TSD were soaked in double-distilled water (the mass ratio of medication on the water was equal to $1: 8$ ) for more than 2 hours. The mixture of medications was decocted for $50 \mathrm{~min}$. The resulting decoction was filtered with gauze and the filtrates were centrifugated at $3000 \mathrm{r} / \mathrm{min} 4{ }^{\circ} \mathrm{C}$ for 
$10 \mathrm{~min}$. The supernatants of the two parts of decoctions were combined and then lyophilized into powder and stored at $-80^{\circ} \mathrm{C}$ before medication administrations.

To ensure the homogeneity of TSD used during the two-week intervention, the internal standard Lnorvaline $(1 \mathrm{mg} / \mathrm{L}$, relative standard divergence $(R S D)=0.09)$ added in the prepared TSD samples was evaluated every three days before the next gavage (shown in Table S-1). The sample preparation and the determination of the internal standard based on gas chromatography coupled with mass spectrometry (GC-MS) have been described in the supplementary data. To authenticate all the herbs bought in the pharmacy, comparisons between the standard products of the six herbs included in TSD and the medications applied to feed the rats were done with the non-targeted metabolomic analyses. Figure S-1 shows an example of the comparison between the metabolic fingerprint of an extracted Radix Paeoniae Alba sample and that of the standard product. The instrumental conditions are also noted in the supplementary material.

\subsection{Animal modeling and treatment}

All the rats underwent an adaptive feeding one week before further treatments. They were randomly divided into 6 groups as follows: the blank control group (B), the model group (M); the model group treated with fluoxetine ( $F X, 10 \mathrm{mg} / \mathrm{kg})$, and the model group treated with TSD $(2.5(T L) \otimes 5(T M)$ and $10 \mathrm{~g} / \mathrm{kg}$ $(\mathrm{TH})$ ). Except for the blank controls, the DD was imitated by the induction of chronic unpredictable mild stress (CUMS) stimulations[19] in modeled rats. The operations included water fasting, reversal of day and night, tilting, dampness, crowding, water fasting and dampness (24h), and ice water stimulation $\left(4^{\circ} \mathrm{C}, 5 \mathrm{~min}\right)$ in turn. The modeling groups were repeatedly and randomly stimulated for 35 days. Of the five modeling groups, three groups of CUMS rats were treated with low $(2.5 \mathrm{~g} / \mathrm{kg}$. D), medium $(5 \mathrm{~g} / \mathrm{kg}$. D), and high $(10 \mathrm{~g} / \mathrm{kg}$. D) doses of TSD, respectively, while the rats in the FX group were subject to the fluoxetine (positive control, $10 \mathrm{~g} / \mathrm{kg}$. D). Prepared TSD powder and fluoxetine were dissolved in $0.5 \%$ sodium carboxymethyl cellulose (CMC-Na) suspension before medication administrations. The other two groups were meanwhile subject to saline injections.

\subsection{Bodyweight measurements and behavioral tests}

The bodyweight measurement and the behavioral tests were first performed before medication interventions to verify the success of the model establishment. The same tests were repeated after a twoweek medication therapy to observe the effect of TSD on depression-like symptoms. Open-field tests (OFT) were carried out with light alteration as described previously [20]. Briefly, the OFT apparatus was a black wooden box $(100 \mathrm{~cm}$ long $\times 100 \mathrm{~cm}$ wide $\times 50 \mathrm{~cm}$ high). The floor of an arena was divided into 25 equal-size squares by white lines. Groups of rats were placed individually into the corner of the apparatus back to the wall and were allowed to explore freely for $5 \mathrm{~min}$. A minimal amount of light (60 Ix) was used to avoid anxiety behavior. The apparatus was cleaned with $90 \%$ ethanol and dried before the next test to remove the smell from the former animal. The number of rearing, grooming, and defecation, and standing in the center were manually recorded by trained observers blind to the experiment. Forced swimming tests (FST) were conducted according to our previous work [21]. All the rats were separated and individually 
put into a transparent glass cylinder ( $60 \mathrm{~cm}$ tall $\times 25 \mathrm{~cm}$ inner diameter) filled with water. The water temperature was maintained at $25 \pm 1^{\circ} \mathrm{C}$ during the experiments. Immobility time was defined as the period when there was a lack of body motion with only the small necessary movements such as taking a breath above the water surface. The test for each rat lasted 6 minutes, in which the immobility time was measured on the final 5 min.

\subsection{Sample collections from rats}

Twenty-four hours after the final behavioral tests, the animals were sacrificed immediately under anesthesia. Rat blood was sampled from the abdominal aorta. The serum was kept after centrifugation at $3000 \mathrm{r} / \mathrm{min}$ and $4^{\circ} \mathrm{C}$ for $15 \mathrm{~min}$. Brain tissues of the rats were meanwhile retained in which the hippocampi were dissected and collected in an ice bath. Hippocampus samples were subsequently snapfrozen by liquid nitrogen and subsequently stored at $-80^{\circ} \mathrm{C}$ until analysis.

\subsection{Serum sample preparations}

For the metabolomic analyses, samples were first thawed at room temperature. A series of metabolite extraction and derivatization reactions were performed with $100 \mu \mathrm{L}$ of each serum sample from each rat following the same method applied in our previous work [22]. An equal volume of each collected sample was taken out to mix up the quality control (QC) pool. The QC samples were prepared in the same way as other real samples during the metabolite extraction and derivatization reactions. The QC samples were used to serve for balancing the instrument and for estimating the stability of the analyses. In the sequence of GC-MS analyses, the QC sample was randomly inserted after every 5 real samples.

\subsection{GC-MS conditions}

A Shimadzu GC-MS 2010 was used for the determination of the metabolic profiling in each sample. The GC-MS condition referred to our previous work [22] except for the gradient temperature program. For this current work, the gradient temperature program was set as follows: the inlet temperature was set at $300^{\circ} \mathrm{C}$, the interface transfer temperature was $280^{\circ} \mathrm{C}$, and the ion source temperature was $230^{\circ} \mathrm{C}$. The column flow was $1.2 \mathrm{~mL} \cdot \mathrm{min}^{-1}$, and the split ratio was set as $10: 1$. The event time was set to $0.2 \mathrm{~s}$. Ions with their mass-to-charge ratios ranging from 50 to $600 \mathrm{~m} / \mathrm{z}$ were detected under full-scan mode. The injection volume was $1 \mu \mathrm{L}$ for each data acquisition. The gradient temperature program was set as follows: initial column temperature was set to $70^{\circ} \mathrm{C}$ and was maintained for $3 \mathrm{~min}$. The temperature was then increased to $200^{\circ} \mathrm{C}$ with a gradient of $3^{\circ} \mathrm{C} \cdot \mathrm{min}^{-1}$ and was then held for $5 \mathrm{~min}$. A gradient of $5^{\circ} \mathrm{C} \cdot \mathrm{min}^{-1}$ was applied while the temperature raised to $300^{\circ} \mathrm{C}$ and the column temperature was finally maintained for $3 \mathrm{~min}$.

\subsection{Data processing and data analysis for the metabolic profiling}

One of the initially obtained total ion chromatography diagrams has been shown in Figure S-2. The acquired data were first processed with the steps of ion peak alignment, deconvolution, normalization as described in extenso previously [23]. Briefly, normalized peak areas were used for the relative 
quantification for each ion peak. The annotation was done with the NIST database (version 2019) and by comparing it to the spectra of the standard substance. The screening of data was conducted within the QC samples in light of the relative standard derivation (RSD) and the ratio of signal on noise $(\mathrm{S} / \mathrm{N})$ of each ion peak. Those metabolites with their S/N inferior to 3 or with their RSD superior to $30 \%$ across all the QC samples were removed. After the pre-processing and the screening, the data set was subject to Metaboanalyst software (Version 5.0, www.metaboanalyst.ca) for further analyses [24]. The online opensource software assumes the analyses such as principal component analysis (PCA), orthogonal projection to latent structures-discriminatory analysis (OPLS-DA), and metabolic pathway analysis. The pvalue of student's T-test between compared groups was calculated by Graphpad Prism software (version 9.0, San Diego, USA).

\subsection{ELISA assays}

Serum serotonin and Arg I were evaluated by ELISA kits produced by Abcam Company (Cambridge, UK) according to the instructions provided by the manufacturer. The concentration of serotonin was measured at $405 \mathrm{~nm}$ while the concentration of serum Arg I in rats was measured at $450 \mathrm{~nm}$.

\subsection{Western Blot Assays}

Tissue samples were homogenized in radioimmunoprecipitation assay (RIPA) buffer (50 mM Tris- $\mathrm{HCl}, \mathrm{pH}$ 7.4, $0.1 \%$ SDS, $1 \%$ NP-40, 0.25\% sodium deoxycholate, 150 mM NaCl, 1 mM EDTA, 1 mM EGTA, and 1 mM $\mathrm{Na}_{3} \mathrm{VO}_{4}$ ). Before homogenization, a protease inhibitor cocktail (Servicebio Technology Co. Ltd) and a phosphatase inhibitor PhosSTOP (Servicebio Technology Co. Ltd) were added. Protein quantitation was conducted using a BCA Protein Concentration Assay Kit (Sparkjade Science Co.Ltd.China). The same quantity (approximately $50 \mu \mathrm{g}$ ) of protein from each sample was loaded and separated by $12 \%$ SDSPAGE and then transferred onto a polyvinylidene difluoride membrane (Amersham Biosciences, UK). The membrane was blocked with $5 \%$ skim milk for $1 \mathrm{~h}$, incubated with antibodies targeting BDNF (1:500; Servicebio Technology Co. Ltd), TrkB (1:500; Servicebio Technology Co. Ltd), CREB (1:1000; Abcam, UK, Cata), ERK1/2 (1:1000; Abcam, UK, Cata), p-ERK1/2 (1:1000; Abcam, UK, Cata) or $\beta$-actin (1:1000; Kangchen Biotechnology, INC, Shanghai, China) at $4^{\circ} \mathrm{C}$ overnight. Next, the membranes were incubated with a horseradish peroxidase-conjugated secondary antibody $(1: 2000)$ at $37^{\circ} \mathrm{C}$ for $2 \mathrm{~h}$. Blots were developed with the Easy Enhanced Chemiluminescence Western Blot Kit (Pierce Biotechnology, Rockford, IL, USA).

The relative expression levels of proteins were determined using a densitometer and were normalized by referring to $\beta$-actin. Image $J$ software $(\mathrm{NIH}, \mathrm{USA})$ was used to quantify relative protein expressions. Oneway analysis of variance (ANOVA) with the Tukey HSD correction was used to determine the significant differences of protein expressions between the groups.

\section{Results}




\subsection{Effect of TSD on depressive behavior in CUMS model rats}

The animal experiment process has been displayed in Fig. 1a. Inhibited anabolism and malnutrition are common symptoms of DD. We, therefore, measured the weights of all the rats before and after medication interventions. General decreases of body weight were observed in CUMS model rats compared with the non-treated controls. Rats treated with $\operatorname{TSD}(2.5,5$, and $10 \mathrm{~g} / \mathrm{kg})$ and fluoxetine $(\mathrm{FX}$, positive control, $10 \mathrm{mg} / \mathrm{kg}$ ) significantly increased their body weights compared with the model group (Fig. 1b). OFT and FST were also performed in accompany with the weight measurements. The first behavioral tests aimed to confirm the success of the model establishment while the repeated actions after medication administrations served to visualize the effect of TSD on depression. Accordingly, model rats before pharmacotherapies presented extended periods of immobility compared with controls in the behavioral tests, showing that the models were successfully established. The episodes of immobility were significantly reduced in the groups with TSD or FX treatments, which confirmed the anti-depressant effect of TSD in rats as shown in Fig. 1c-e.

\subsection{Effect of TSD on the serum metabolome of CUMS rats}

After the samplings of rat serum, we applied the GC-MS-based metabolomics method in the rats to study the metabolic characteristics of each group, especially to have an insight into the metabolic alterations caused by TSD administration. Before data analysis, an assessment of the stability of instrumental data acquisition was conducted with the QC samples. The QC samples were emerged in a PCA by differentiation from the real samples. A clear gather of the QC samples surrounded by the real samples in the PCA shown in Figure S-3, which proved a satisfying reproducibility for the analyses. Consequently, we identified 82 metabolites among the retained 166 ion peaks. MetaboAnalyst 5.0 software was employed to analyze the data corresponding to the refined and normalized peak areas of metabolites. Figure 2 shows differences in the metabolic profiles between different groups by the score plots of PCA and OPLSDA. Concretely, a PCA separating the blank controls from the CUMS model group was shown in Fig. 2a $(P C 1=18 \%, P C 2=13.8 \%)$. The same separation based on OPLS-DA (two components, $R^{2} X=0.511, R^{2} Y=$ $0.993, Q^{2} Y=0.985$ ) also showed clear discrimination between these two groups (Fig. 2b). Aiming to understand the effect of TSD on the metabolome of CUMS rats, another PCA was conducted between the model group (red dots illustrated in Fig. $2 \mathrm{c}, \mathrm{PC} 1=12.3 \%, \mathrm{PC} 2=9.7$ ) and TSD treatment groups with different dosages (presented by blue, cyan, and green dots, respectively). Correspondingly, the model group samples in the PCA were shown to be separated from those of TSD-treated groups. However, unexpectedly, few metabolic differences were present for the model rats fed with different dosages of TSD. Clear discrimination of metabolome was otherwise confirmed between the CUMS rats and overall TSD-treated rats by another OPLS-DA $\left(R^{2} X=0.075, R^{2} Y=0.811, Q^{2} Y=0.588\right.$, Fig. $\left.2 d\right)$. Variable permutation verifications exhibited that the OPLS models were not over-fitting (Figure S-4). Differential metabolites $(p<0.05)$ associated with CUMS modeling and with the effect of TSD intervention are shown in Table S-2. The features which contributed to the separation of $B$ vs. $M$ and the separation of $M$ vs. 
overall TSD-treated rats are equally listed in the Venn diagram (Fig. 3a). Fifteen common discriminants were observed for both the separation of Blank vs. Model and the separation of Model vs TSD treated. Among these key metabolites, urea, and ornithine (Fig. 3b-c) significantly increased in the model group compared with the control group, but significantly decreased in the TSD treatment group. Other primary discriminants comprising four amino acids (AAs), phosphate, citrate acid, lactate, pseudouridine, Myoinositol, and 3-aminoisobutyric acid (3-AIBA). These molecules were found to be rifely decreased in the model rats, but restored in the treated groups, as shown in Fig. 3d-p. An enrichment of primarily varied metabolic pathways was performed along with the determination of metabolic features between groups. As shown in Fig. 3q-r, varied metabolic pathways associated with the depression (comparison between the $\mathrm{M}$ and $\mathrm{S}$ group) were similar to those associated with the effect of TSD on depression (comparison between the $M$ and integrated groups of rats treated with TSD). Notably, arginine-related pathways were determined as primary characteristics in both pathway analyses.

\subsection{Effects of TSD on serum levels of arginase I and serotonin}

Arginase I (Arg I) is the enzyme that contributes to the conversion from arginine to urea and ornithine. We assessed Arg I in the serum of all the groups of rats since consistent enrichments were found in urea and ornithine in CUMS rats but such an effect was alleviated by TSD administration. Our data showed that Arg I was decreased in the model group but re-increased in the TSD-fed groups (Fig. 4a), which was in concert with the above two products of arginase degradation. Meanwhile, considering that increasing evidence shows the enhancement of serotonin by herbal medications, we also tested serum serotonin by another ELISA assay. As shown in Fig. 4b, an obvious decrement of serotonin was observed in the CUMS rats without treatments in comparison to the blank controls. The depletion of serotonin was attenuated in the rats with TSD interventions. Such effect of TSD on serotonin was found to be dose-dependent. There was no surprise even a more adequate recovery of serum serotonin was found in the CUMS rats treated with FX since 5-HT receptors are known targets of FX.

\subsection{Effects of TSD on BDNF, TrkB, ERK, PERK, and CREB protein expression in the hippocampus}

It was previously reviewed by Caldwell et al. that CAMP response element-binding protein (CREB) was involved in the transcription of Arg I [25]. CREB is modulated by the BDNF-TrkB- ERK signaling cascade. Strikingly, we noticed that the BDNF-CREB axis was tightly related to the pathology of depression [26]. Therefore, to determine whether the effect of TSD on Arg I release was due to the regulation on the BDNFCREB axis, we evaluated the related target proteins in the hippocampus of rats by Western blot analysis (Fig. 5a). The results displayed the expression levels of BDNF and TrkB proteins were significantly downregulated compared with the normal group (BDNF: $(F(5,17)=5.025, P<0.05)$; TrkB: $(F(5,17)=$ 33.093, $\mathrm{P}<0.001)$; CREB: $(\mathrm{F}(5,17)=35.045, \mathrm{P}<0.001))$, and the effect was effectively reversed by TSD $(2.5,5$ and $10 \mathrm{~g} / \mathrm{kg})$ treatment. Also, the expression of ERK1/2 were decreased by CUMS model $(F(5,17)$ $=15.190, \mathrm{P}<0.01)$, TSD treatment can increase the phosphorylation level of hippocampal ERK1/2. These 
results suggested that the TSD might exert its antidepressant effect by inhibiting arginase, which was related to the up-regulation of the BDNF/ERK/CREB signaling pathway. Together, a schema summarizing possible protein targets of TSD belonging to the BDNF-CREB-Arg I axis is shown in Fig. $5 \mathrm{~b}$.

\section{Discussion}

The incidence of DD is increasing globally in recent years, which has been a worldwide public health problem. Safe and effective anti-depression drug development is always required. Recent studies have shown that ethnomedicine is a great treasure for the development of effective drugs treating depression [27]. In particular, some formulas of CTM have been reported to be useful to improve DD [28]. To our best known, here we first report the effect of TSD on depression.

In this current study, depression-like symptoms were induced with CUMS that is a modeling method well imitating the occurrence and pathogenesis of human depression [29]. After the modeling within rats, results of behavioral experiments exhibit that such a modeling method is reliable. Also, those rats treated with TSD emerge significant increases of time of immobility in the behavioral experiments. The improvements of depression-like symptoms in rats are to some extent even better than the effect of fluoxetine according to our data. A further investigation based on metabolomic techniques is conducted to provide a better understanding of aberrant metabolic variations associated with CUMS and of the pharmacological effect of TSD on DD. Dysregulations of two urea cycle intermediates, urea, and ornithine, are observed to be primary differential factors for the discrimination between rats with and without TSD gavage, and between the blank group and the model group. The increases of these two metabolites in the serum of model group rats indicate enhanced arginase degradation, which is found to be ameliorated after the intervention of TSD administration. The effect is further demonstrated by the results of serum Arg I, which shows that the variations of serum arginase I are in line with those of urea and ornithine. It is documented previously that the increase of arginase in the blood is associated with the occurrence of depression [30]. Interestingly, the level of arginase I is also regarded to be predicable for liver and heart injury [31]. Correspondingly, TSD was widely used for the treatment of heart diseases and was documented to be effective for improving liver injury [32,33]. Therefore, the restoration of arginase after the dosing of TSD may also indicate reversals of organ injuries caused by the CUMS modeling. In addition, the up-regulation of arginase is also related to immune defection and aberrant responses to inflammation [34]. The reversal of arginase disorder within TSD-treated rats may be partly owing to the anti-inflammatory effect of TSD, which is documented in another study [35].

General declines of AAs (including glycine, alanine, proline, phenylalanine, glutamate, lysine, and tyrosine) in the serum are remarkable features of CUMS. After treated with TSD, levels of most AAs recover in the serum of rats. First, decreases in AAs suggest a malnutrition situation of CUMS rats, which is reported previously [36]. Such a symptom is evidenced by the weight losses (Fig. 1a) in the model group. The impaired anabolic metabolism can be partly reflected by the lower levels of energetic metabolites such as citrate and lactate in CUMS rats, compared to other groups. Notably, common discriminants between M vs. $B$ and $M$ vs. TSD-treated like alanine and glutamate are AAs involved in the anaplerotic reactions from 
AA to the TCA cycle, which is the core metabolic pathway of energy supply. Second, ameliorations in the defective Tyr/Phe pathway are also found in the TSD-treated rats which is in accordance with the study by Liu et al [37]. Finally, glycine as well as glutamate which can be converted into GABA are associated with neurotransmitters. Thus, besides the diminished secretion of dopamine and 5-HT, depressive appearances of CUMS rats can be strongly attributed to the inhibited transmitter synthesis.

Other common discriminant metabolites between $\mathrm{M}$ vs. B and M vs. TSD include 3-AIB, pseudouridine, and 3-HB. 3-AIB is known as a critical synthetic precursor of AAs, its variation pattern is in line with most serum AAs in rats. Peseudouridine was reported recently as a potential biomarker of post-stroke depression [38]. 3-HB is an end-product of beta-oxidation in fatty acids. Decrease 3-HB in the CUMS rats is consistent with the diminished serum fatty acids such as propanoic acid in comparison with the blank controls, and with lower levels of arachidic acid found in the comparison between CUMS and non-treated rats.

Our data also suggest that the dramatic dysregulation of Arg I in depressive rats is involved in the inhibited BDNF/CREB signaling and is reversible after the intervention of TSD. Evidence support that BDNF as well as its membranal receptor in the brain, TrkB, in the hippocampus is implicated in both the pathogenesis of depression and antidepressant actions [39]. Several studies have shown that medications targeting the BDNF-TrkB-CREB axis improve depression-like symptoms in animal models [40]. Interestingly, a putative compound hydroxysafflor $A$ issued from safflower is reported to up-regulate BDNF expression [41]. Similar results are available in another recent work showing the effect of safflower yellow, a drug extracted from safflower, on the activation of BDNF/TrkB/ERK signaling [42]. Alternatively, ethanol extract of Radix Rehmanniae praeparata also helps to enhance BDNF levels in the serum of CUMS rats [43]. Other potential compounds contained in TSD involving depression therapy encompass albiflorin from Radix Paeoniae alba [44], ferulic acid from both Rhizoma Chuanxiong and Angelica Sinensis [45], and ligustrazine from Rhizoma Chuanxiong. Remarkably, ferulic acid can not only increase BNDF expression but also reduce oxidative stress induced by chronic corticosterone stimulation in mice [46]. Given that Arg I is a pivotal marker of NO production, with hints that the effect of TSD on depression may be due to the anti-inflammatory effect. Ligustrazine was also reported to attenuate inflammation and oxidative stress in rats recently [47]. Furthermore, our findings support that the effect of TSD on DD is linked to the regulation of serotonin secretion. Emerging data show close interactions between BDNF and serotonin. Some studies suggested that BDNF was the target of serotonin [48]. However, the reverse regulation on serotonin receptor 5-HT1A by BDNF expression was also reported by Homberg et al. [49]. Together, our data imply that the actions of TSD on BDNF and serotonin are interdependent.

Our work does have some limitations. First, it remains unclear that what is the most effective composition or compound in TSD improving CUMS-induced depressive symptoms. Second, even though obvious metabolic differences and key discriminants are revealed between CUMS rats and TSD-treated ones, the variation patterns of the discriminants are not dose-dependent according to our data. This issue is supposed to be ascribed to the imitated sample size. Further study will be an investigation of antidepressant effects of the six component medications of TSD within a larger number of samples. 
To summarize, our findings display that the TCM formula TSD can be a useful complementary therapy for depression management. The underlying mechanism behind the efficacy of TSD on DD should be multi-targeted, which can be related to the enhancement of neural transmitters such as glycine and serotonin, as well as to the improvement of disorders on the BNDF-CREB-Arg I axis.

\section{Abbreviations}

Tao-Hong-Si-Wu decoction (TSD)

depression disorders (DD)

chronic unpredictable mild stress (CUMS)

A gas-chromatography coupled with mass spectrometry (GC-MS)

$\operatorname{arginase~I~(Arg~I)~}$

hypothalamic-pituitary-adrenal (HPA)

Chinese traditional medicine (CTM)

$\mathrm{N}$-methyl-N-trimethylsilyltrifluoroacetamide (MSTFA)

relative standard divergence (RSD)

the blank control group (B)

the model group (M)

the model group treated with fluoxetine (FX)

CUMS rats treated with a low dose of TSD (TL)

CUMS rats treated with a medium dose of TSD (TM)

CUMS rats treated with a high dose of TSD $(\mathrm{TH})$

sodium carboxymethyl cellulose (CMC - Na)

Open-field tests (OFT)

Forced swimming test (FST)

quality control (QC)

radioimmunoprecipitation assay (RIPA) 
analysis of variance (ANOVA)

signal on noise $(\mathrm{S} / \mathrm{N})$

principal component analysis (PCA)

orthogonal projection to latent structures-discriminatory analysis (OPLS-DA)

amino acids (AAs)

3-aminoisobutyric acid (3-AIBA)

cAMP response element-binding protein (CREB)

Brain-derived neurotrophic factor (BDNF)

Tropomyosin receptor kinase B (TrkB)

Extracellular signal-regulated kinases (ERK)

\section{Declarations}

\section{Ethics approval and consent to participate}

All the procedures in the animal experiments followed the "Principles of Laboratory Animal Care" (NIH Publications No. 85-23, revised 1996) and complied with the rules of the Ethics Committee and the Animal Experimental Committee of Anhui Medical University, China.

\section{Consent for publication}

Not applicable.

\section{Availability of data and materials}

All the data generated or analyzed during this study are included in the manuscript and the supplementary data.

\section{Competing interests}

The authors declare that there is no conflict of interest.

\section{Funding}

The study is supported by the National Natural Science Foundation of China (0601055205), and by Anhui Natural Science Foundation (2008085QH364), and by the funding of Anhui Medical University (XJ201729, 2020xkjT019). 


\section{Authors' Contributions}

Xiaoping Zhang: Conceptualization, Writing-original draft, Formal analysis, Methodology;

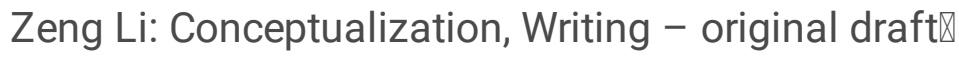

Chuanpu Shen: Conceptualization, Investigation;

Jinzhi He: Writing - original draft, Investigation;

Longfei Wang: Data curation, Validation, Methodology

Lei Di: Data curation, Methodology;

Bin Rui: Data curation, Validation ;

Ning Li: Conceptualization, Project administration $\rrbracket$

Zhicheng Liu: Conceptualization, Data curation, Writing-original draft, Validation, Project administration;

All the authors contributed to the Review \& Editing.

\section{Acknowledgment}

The authors appreciate constructive advice on the study design from Pr. Jinfang Ge and Pr. Rong Li. We also need to thank Mr. Lan Zhang and Mr. Junlong Li for their help on animal experiences.

\section{References}

1. Moussavi S, Chatterji S, Verdes E, Tandon A, Patel V, Ustun B. Depression, chronic diseases, and decrements in health: results from the World Health Surveys. Lancet. 2007;370:851-8. 10.1016/s0140-6736(07)61415-9.

2. Friedrich MJ. Depression is the leading cause of disability around the world. Jama. 2017;317:15177. 10.1001/jama.2017.3826.

3. Malhi GS, Mann JJ: Depression. The Lancet. 2018;392:2299-312. 10.1016/s0140-6736(18)31948-2.

4. Li C, Huang J, Cheng YC, Zhang YW. Traditional Chinese Medicine in Depression Treatment: From Molecules to Systems. Front Pharmacol. 2020;11:586. 10.3389/fphar.2020.00586.

5. Zhu Y, Duan X, Cheng X, Cheng X, Li X, Zhang L, Liu P, Su S, Duan JA, Dong TT, et al. Kai-Xin-San, a standardized traditional Chinese medicine formula, up-regulates the expressions of synaptic proteins on hippocampus of chronic mild stress induced depressive rats and primary cultured rat hippocampal neuron. J Ethnopharmacol. 2016;193:423-32. 10.1016/j.jep.2016.09.037.

6. Sun KH, Jin Y, Mei ZG, Feng ZT, Liu JR, Cao MQ, Wu ZZ. Antidepressant-Like Effects of Chaihu Shugan Powder on Rats Exposed to Chronic Unpredictable Mild Stress through Inhibition of 
Endoplasmic Reticulum Stress-Induced Apoptosis. Chin J Integr Med. 2021;27:353-60.

10.1007/s11655-020-3228-y.

7. Li JM, Kong LD, Wang YM, Cheng CH, Zhang WY, Tan WZ. Behavioral and biochemical studies on chronic mild stress models in rats treated with a Chinese traditional prescription Banxia-houpu decoction. Life Sci. 2003;74:55-73. 10.1016/j.lfs.2003.06.030.

8. Chi X, Wang S, Baloch Z, Zhang H, Li X, Zhang Z, Zhang H, Dong Z, Lu Y, Yu H. Research progress on classical traditional Chinese medicine formula Lily Bulb and Rehmannia Decoction in the treatment of depression. Biomed Pharmacother. 2019;112:108616. 10.1016/j.biopha.2019.108616.

9. Wang Y-S, Shen C-Y, Jiang J-G. Antidepressant active ingredients from herbs and nutraceuticals used in TCM: pharmacological mechanisms and prospects for drug discovery. Pharmacological research. 2019;150:104520. 10.1016/j.phrs.2019.104520.

10. Ahmed T, Vasiliadis HM. Global cognition modifies the relationship between anemia and depression in old age: A longitudinal analysis of the IMIAS Study. Arch Gerontol Geriatr. 2021;94:104342. 10.1016/j.archger.2021.104342.

11. Shen F, Song Z, Xie P, Li L, Wang B, Peng D, Zhu G. Polygonatum sibiricum polysaccharide prevents depression-like behaviors by reducing oxidative stress, inflammation, and cellular and synaptic damage. J Ethnopharmacol. 2021;275:114164. 10.1016/j.jep.2021.114164.

12. Kostev K, Jacob L, Kalder M. Risk of depression, anxiety, and adjustment disorders in women with a suspected but unconfirmed diagnosis of breast or genital organ cancer in Germany. Cancer Causes Control. 2017;28:1021-6. 10.1007/s10552-017-0948-1.

13. Li L, Yang N, Nin L, Zhao Z, Chen L, Yu J, Jiang Z, Zhong Z, Zeng D, Qi H. Chinese herbal medicine formula Tao Hong Si Wu decoction protects against cerebral ischemia-reperfusion injury via PI3K/Akt and the Nrf2 signaling pathway. Journal of natural medicines. 2015;69:76-85. 10.1007/s11418-014-0865-5.

14. Zhao Z-X, Fu J, Ma S-R, Peng R, Yu J-B, Cong L, Pan L-B, Zhang Z-G, Tian H, Che C-T. Gut-brain axis metabolic pathway regulates antidepressant efficacy of albiflorin. Theranostics. 2018;8:5945. $10.7150 /$ thno. 28068 .

15. Chen J, Lin D, Zhang C, Li G, Zhang N, Ruan L, Yan Q, Li J, Yu X, Xie X. Antidepressant-like effects of ferulic acid: involvement of serotonergic and norepinergic systems. Metabolic brain disease. 2015;30:129-36. 10.1007/s11011-014-9635-z.

16. Wang CF, Cai XR, Chi YN, Miao XY, Yang JY, Xiao BK, Huang RQ. Analgesic Activity of Jin Ling Zi Powder and Its Single Herbs: A Serum Metabonomics Study. Chin J Integr Med. 2021. 10.1007/s11655-021-3277-x.

17. Wang XJ, Ren JL, Zhang AH, Sun H, Yan GL, Han Y, Liu L. Novel applications of mass spectrometrybased metabolomics in herbal medicines and its active ingredients: current evidence. Mass Spectrom Rev. 2019;38:380-402. 10.1002/mas.21589.

18. Tuyiringire N, Tusubira D, Munyampundu J-P, Tolo CU, Muvunyi CM, Ogwang PE. Application of metabolomics to drug discovery and understanding the mechanisms of action of medicinal plants 
with anti-tuberculosis activity. Clinical translational medicine. 2018;7:1-12. 10.1186/s40169-0180208-3.

19. Kim YK, Na KS, Shin KH, Jung HY, Choi SH, Kim JB. Cytokine imbalance in the pathophysiology of major depressive disorder. Prog Neuropsychopharmacol Biol Psychiatry. 2007;31:1044-53. 10.1016/j.pnpbp.2007.03.004.

20. Liu L, Dong Y, Shan X, Li L, Xia B, Wang H. Anti-Depressive Effectiveness of Baicalin In Vitro and In Vivo. Molecules 2019, 24. 10.3390/molecules24020326.

21. Zhang L, Yang Y, Di L, Li JL, Li N. Erxian decoction, a famous Chinese medicine formula, antagonizes corticosterone-induced injury in PC12 cells, and improves depression-like behaviours in mice. Pharm Biol. 2020;58:498-509. 10.1080/13880209.2020.1765812.

22. Liu Z, Nahon P, Li Z, Yin P, Li Y, Amathieu R, Ganne-Carrié N, Ziol M, Sellier N, Seror O. Determination of candidate metabolite biomarkers associated with recurrence of HCV-related hepatocellular carcinoma. Oncotarget. 2018;9:6245. 10.18632/oncotarget.23500.

23. Liu Z, Zhang J, Zhang X, Shen C, Yin L, Zhu Y, Li N, Chen F. Metabolic and Inorganic Elemental Profiling Analysis of Tortoise Shell for the Identification of Tortoise Strain. Food Anal Methods. 2020. 10.1007/s12161-020-01908-y.

24. Pang Z, Chong J, Zhou G, de Lima Morais DA, Chang L, Barrette M, Gauthier C, Jacques P-É, Li S, Xia J. MetaboAnalyst 5.0: narrowing the gap between raw spectra and functional insights. Nucleic acids research. 2021. 10.1093/nar/gkab382.

25. Caldwell RW, Rodriguez PC, Toque HA, Narayanan SP, Caldwell RB. Arginase: A Multifaceted Enzyme Important in Health and Disease. Physiol Rev. 2018;98:641-65. 10.1152/physrev.00037.2016.

26. Wang H, Zhang Y, Li H, Zeng W, Qiao M. Shuyu capsules relieve liver-qi depression by regulating ERKCREB-BDNF signal pathway in central nervous system of rat. Exp Ther Med. 2017;14:4831-8. 10.3892/etm.2017.5125.

27. Shao J, Zhou L, Shao T, Ding M, Jin Z. Effectiveness and safety of the Xuefu Zhuyu Tang for poststroke depression: A systematic review and meta-analysis. European Journal of Integrative Medicine 2020, 37. 10.1016/j.eujim.2020.101150.

28. Zhang Y-W, Cheng Y-C. Challenge and prospect of traditional chinese medicine in depression treatment. Front NeuroSci. 2019;13:190. 10.3389/fnins.2019.00190.

29. Geng J, Liu J, Yuan X, Liu W, Guo W. Andrographolide triggers autophagy-mediated inflammation inhibition and attenuates chronic unpredictable mild stress (CUMS)-induced depressive-like behavior in mice. Toxicol Appl Pharmacol. 2019;379:114688. 10.1016/j.taap.2019.114688.

30. Cao B, Deng R, Wang D, Li L, Ren Z, Xu L, Gao X. Association between arginine catabolism and major depressive disorder: A protocol for the systematic review and meta-analysis of metabolic pathway. Medicine. 2020;99:e21068. 10.1097/MD.0000000000021068.

31. Sims CA, Yuxia G, Singh K, Werlin EC, Reilly PM, Baur JA. Supplemental arginine vasopressin during the resuscitation of severe hemorrhagic shock preserves renal mitochondrial function. PLoS One. 2017;12:e0186339. 10.1371/journal.pone.0186339. 
32. Xi S, Shi M, Jiang X, Minuk GY, Cheng Y, Peng Y, Gong Y, Xu Y, Wang X, Yang J. The effects of TaoHong-Si-Wu on hepatic necroinflammatory activity and fibrosis in a murine model of chronic liver disease. J Ethnopharmacol. 2016;180:28-36. 10.1016/j.jep.2016.01.030.

33. Tao T, He T, Mao H, Wu X, Liu X. Non-Targeted Metabolomic Profiling of Coronary Heart Disease Patients With Taohong Siwu Decoction Treatment. Front Pharmacol. 2020;11:651. 10.3389/fphar.2020.00651.

34. Kim E, Jang J, Park JG, Kim KH, Yoon K, Yoo BC, Cho JY. Protein Arginine Methyltransferase 1 (PRMT1) Selective Inhibitor, TC-E 5003, Has Anti-Inflammatory Properties in TLR4 Signaling. Int J Mol Sci 2020, 21. 10.3390/ijms21093058.

35. Yuan G, Han A, Wu J, Lu Y, Zhang D, Sun Y, Zhang J, Zhao M, Zhang B, Cui X. Bao Yuan decoction and Tao Hong Si Wu decoction improve lung structural remodeling in a rat model of myocardial infarction: possible involvement of suppression of inflammation and fibrosis and regulation of the TGF- $\beta 1 /$ Smad3 and NF-KB pathways. Bioscience trends. 2018;12:491-501. $10.5582 /$ bst.2018.01242.

36. Tan YF, Liao ZL, Qiu YJ, Zhu JP, Yu EY. Possible involvement of L-arginine-nitric oxide (NO)-cyclic guanosine monophosphate (cGMP) signaling pathway in the antidepressant-like effect of Wuling mycelia powder in rat. Biomed Pharmacother. 2016;78:60-5. 10.1016/j.biopha.2015.12.016.

37. Liu CC, Wu YF, Feng GM, Gao XX, Zhou YZ, Hou WJ, Qin XM, Du GH, Tian JS. Plasma-metabolitebiomarkers for the therapeutic response in depressed patients by the traditional Chinese medicine formula Xiaoyaosan: A (1)H NMR-based metabolomics approach. J Affect Disord. 2015;185:15663. 10.1016/j.jad.2015.05.005.

38. Cho Y, Hwang H, Rahman MA, Chung C, Rhim H. Elevated O-GlcNAcylation induces an antidepressant-like phenotype and decreased inhibitory transmission in medial prefrontal cortex. Sci Rep. 2020;10:6924. 10.1038/s41598-020-63819-6.

39. $\mathrm{Yu} \mathrm{H}, \mathrm{Chen} \mathrm{ZY}$. The role of BDNF in depression on the basis of its location in the neural circuitry. Acta Pharmacol Sin. 2011;32:3-11. 10.1038/aps.2010.184.

40. Lee YJ, Kim HR, Lee CY, Hyun SA, Ko MY, Lee BS, Hwang DY, Ka M. 2-Phenylethylamine (PEA) Ameliorates Corticosterone-Induced Depression-Like Phenotype via the BDNF/TrkB/CREB Signaling Pathway. Int J Mol Sci 2020, 21. 10.3390/ijms21239103.

41. Xing M, Sun Q, Wang Y, Cheng Y, Zhang N. Hydroxysafflor yellow A increases BDNF and NMDARs in the hippocampus in a vascular dementia rat model. Brain research. 2016;1642:419-25. j.brainres.2016.04.030.

42. Pang J, Hou J, Zhou Z, Ren M, Mo Y, Yang G, Qu Z, Hu Y. Safflower Yellow Improves Synaptic Plasticity in APP/PS1 Mice by Regulating Microglia Activation Phenotypes and BDNF/TrkB/ERK Signaling Pathway. Neuromolecular Med. 2020;22:341-58. 10.1007/s12017-020-08591-6.

43. Wang JM, Pei LX, Zhang YY, Cheng YX, Niu CL, Cui Y, Feng WS, Wang GF. Ethanol extract of Rehmannia glutinosa exerts antidepressant-like effects on a rat chronic unpredictable mild stress 
model by involving monoamines and BDNF. Metab Brain Dis. 2018;33:885-92. 10.1007/s11011018-0202-x.

44. Wang YL, Wang JX, Hu XX, Chen L, Qiu ZK, Zhao N, Yu ZD, Sun SZ, Xu YY, Guo Y, et al. Antidepressant-like effects of albiflorin extracted from Radix paeoniae Alba. J Ethnopharmacol. 2016;179:9-15. 10.1016/j.jep.2015.12.029.

45. Liu Y-M, Hu C-Y, Shen J-D, Wu S-H, Li Y-C, Yi L-T. Elevation of synaptic protein is associated with the antidepressant-like effects of ferulic acid in a chronic model of depression. Physiol Behav. 2017;169:184-8. 10.1016/j.physbeh.2016.12.003.

46. Zeni ALB, Camargo A, Dalmagro AP. Ferulic acid reverses depression-like behavior and oxidative stress induced by chronic corticosterone treatment in mice. Steroids. 2017;125:131-6. j.steroids.2017.07.006.

47. Li Y, Zhu Z, Zhang T, Zhou Y. Ligustrazine attenuates inflammation and oxidative stress in a rat model of arthritis via the Sirt1/NF-KB and Nrf-2/HO-1 pathways. Arch Pharm Res. 2019;42:824-31. 10.1007/s12272-018-1089-0.

48. Martinowich K, Lu B. Interaction between BDNF and serotonin: role in mood disorders. Neuropsychopharmacology. 2008;33:73-83. 10.1038/sj.npp.1301571.

49. Homberg JR, Molteni R, Calabrese F, Riva MA. The serotonin-BDNF duo: developmental implications for the vulnerability to psychopathology. Neurosci Biobehav Rev. 2014;43:35-47. 10.1016/j.neubiorev.2014.03.012.

\section{Figures}




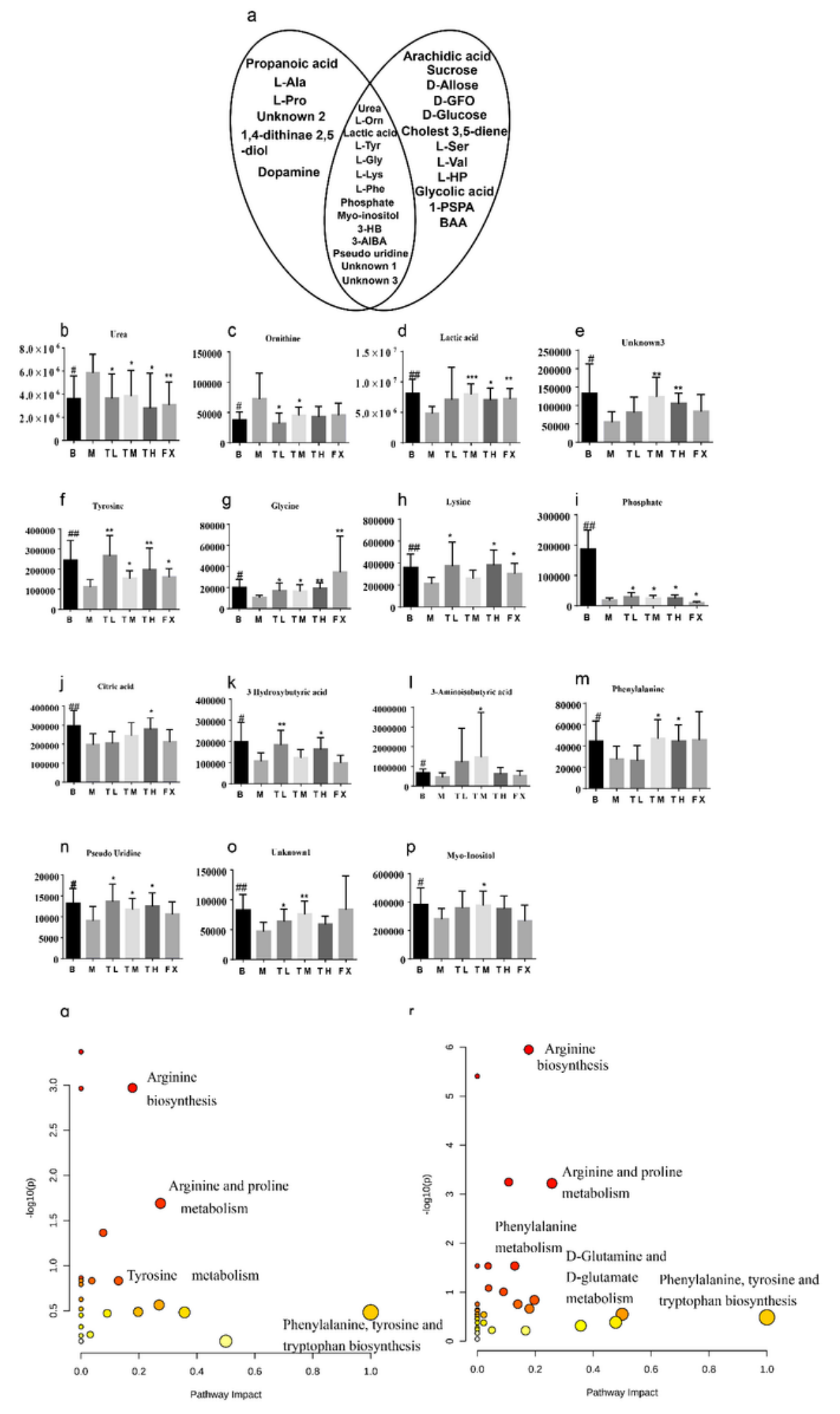

\section{Figure 1}

The comparison of body weight and the findings of behavioral tests before and after dosing of TSD. a: a schema displaying the process of animal experiments; $b$ : Bodyweight changes along with the TSD dosing for the groups of rats; c: The total immobile time of rats recorded in the forced swimming test; d-e: Grooming time and standing time of the rats recorded in the open field test, respectively. 
a

\begin{tabular}{l|c|c|c} 
Adaptation & CUMS & TSD administraton & \\
\hline 7days & 35days & 14days & Days
\end{tabular}

Measurement of Measurement of bodyweight, bodyweight, FST,OFT FST,OFT

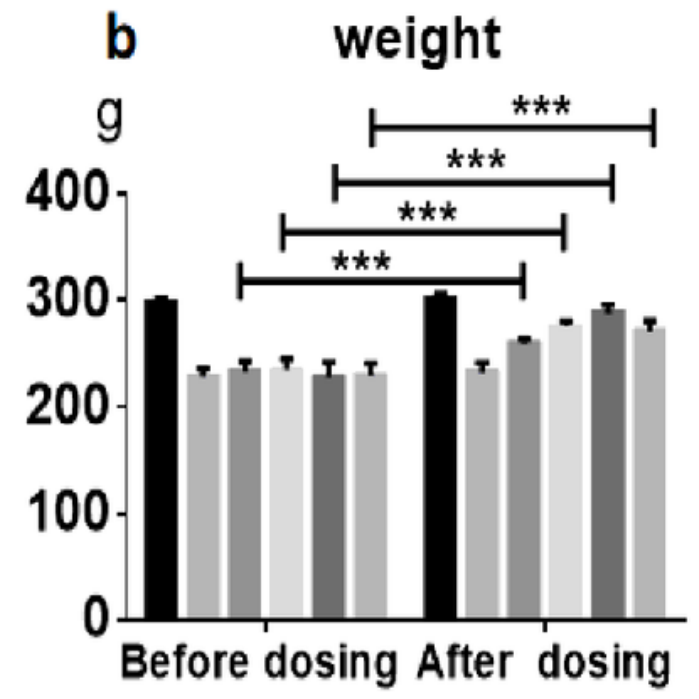

C Swimming test

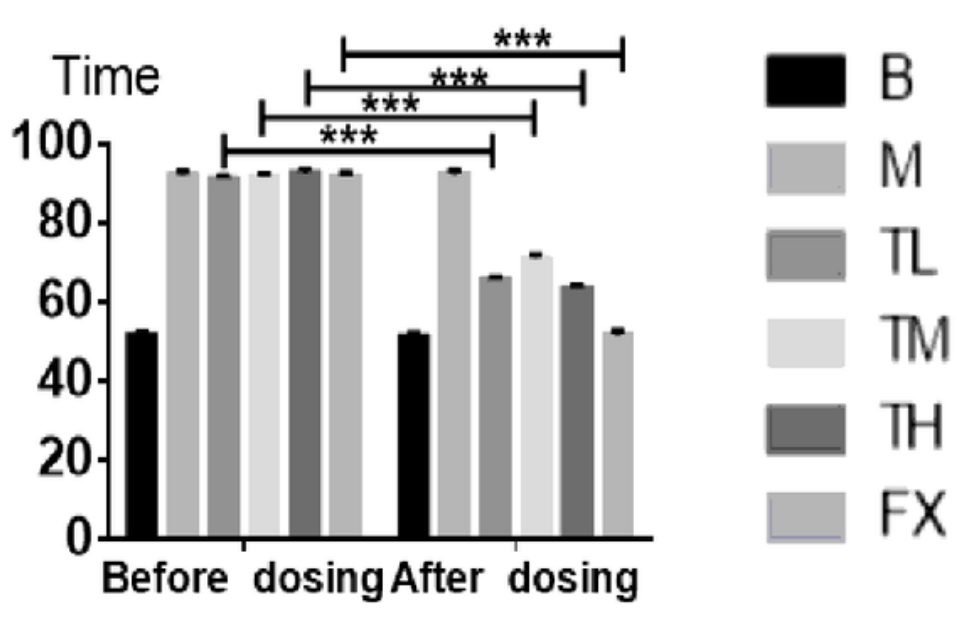

\section{d Grooming time}

Time
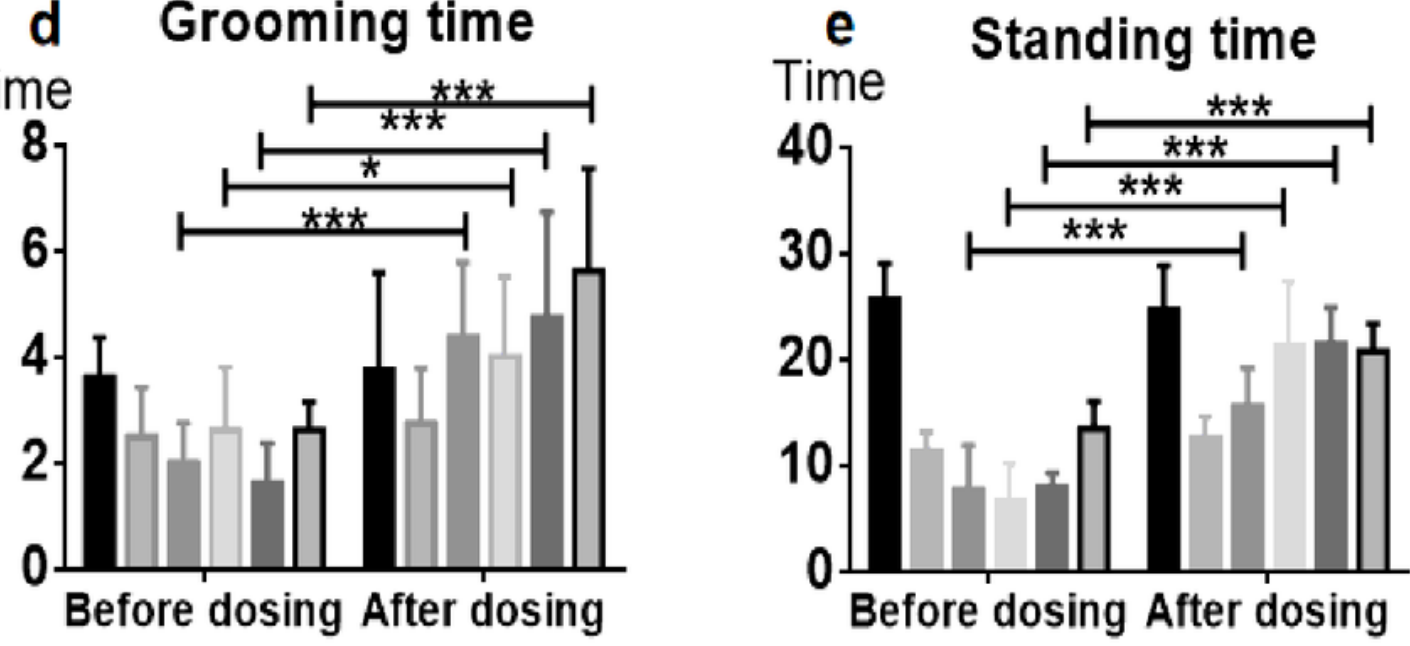

Figure 2

Score plots of PCA and OPLS-DA for the separations of serum metabolome between different groups of rats. a-b: Score plot of PCA and OPLS-DA for the separations between the CUMS model group and the blank group; c: a PCA showing the separation between the CUMS model group and the CUMS rats treated with different dosages of TSD. d: OPLS-DA showing the separation between CUMS model rats and the integration of TSD-treated rats. B: the blank group; M: the model group TL: the model animals treated 
with a low dose of TSD; TM: model animals treated with a medium dose of TSD; TH: rats treated with a high dose of TSD (the same indications are used below); T: TSD-intervened group.
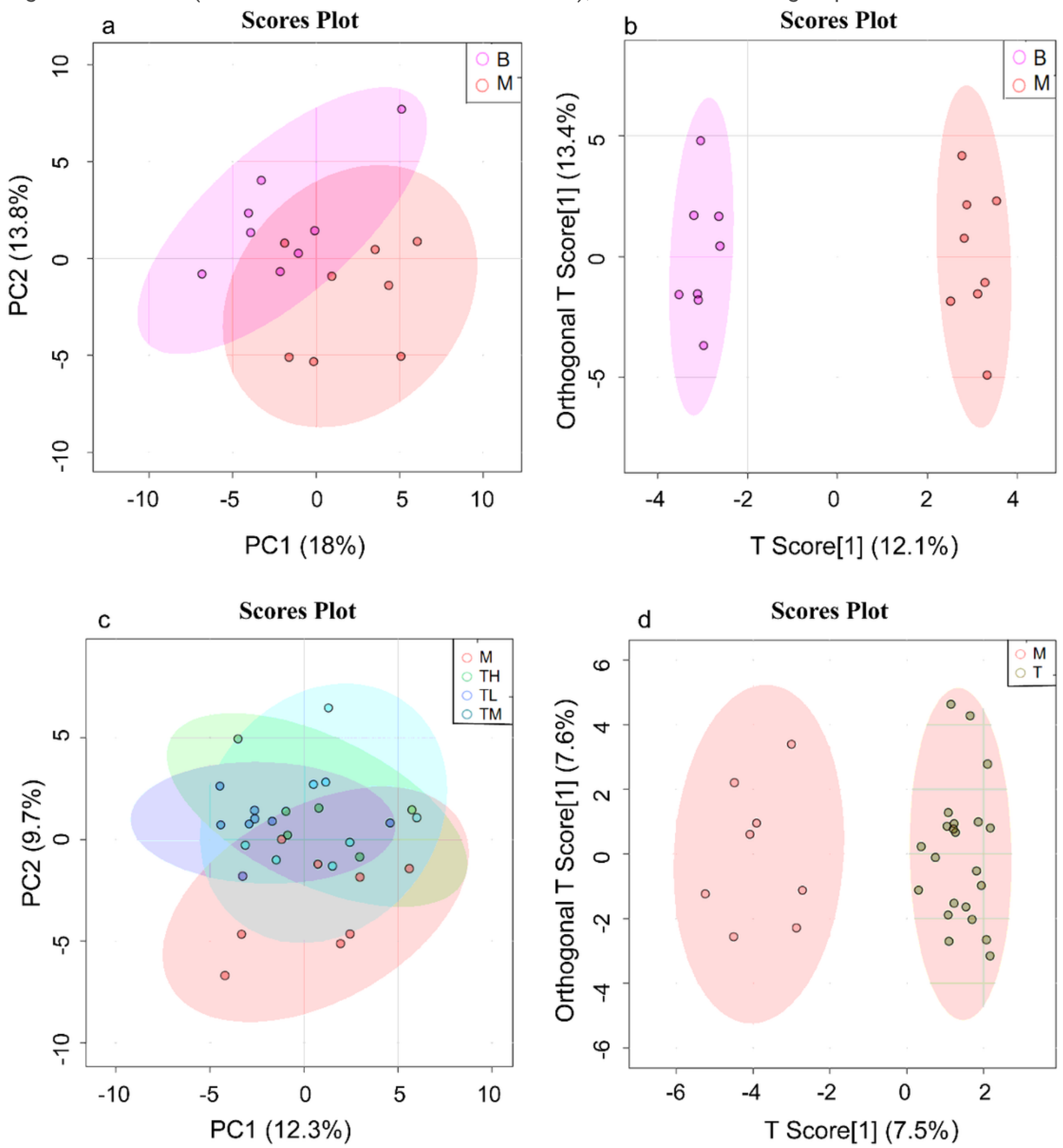

Figure 3

Metabolites with different characteristics between groups of serum metabolism of rats. FX: CUMS rats treated with fluoxetine. a: a Venn diagram showing the differential metabolites associated with CUMS modeling and with the effect of TSD on DD. Metabolites encompassed within the left oval: characteristic 
metabolites involved in the CUMS modeling; metabolites encompassed within the right oval: feature metabolites linked to the effect of TSD on CUMS rats; metabolites included the intersection of the two ovals represent the key metabolites contribute to both the differential analyses $b-p$ : key discriminant metabolites that varied both in the comparison between $B$ vs. $M$ and $M$ vs. TSD; L-Ala: L-alanine; L-Pro: Lproline; L-orn: L-ornithine; L-Tyr: L-Tyrosine; L-Gly: L-glycine; L-Glu: L-Phe: L-phenylalanine; 3-AIBA: 3aminoisobutyric acid; 3-HB: 3-hydroxybutyric acid; L-glutamic acid; L-Ser: L-serine; L-Val: L-valine; L-HP: Lhydroxyproline; D-GFO: Beta-D-galactofuranoside; BAA: genzeneacetic acid; 1-PSPA: 1palmitoyllysophosphatidic acid; q-r: pathway analyses showing the primarily varied metabolic pathways involving the CUMS modeling and the TSD effect, respectively; q: metabolic pathway analyses for the comparison of B vs. M; r: metabolic pathway analysis for the comparison between the model group and the integrated groups of rats treated with TSD.

a

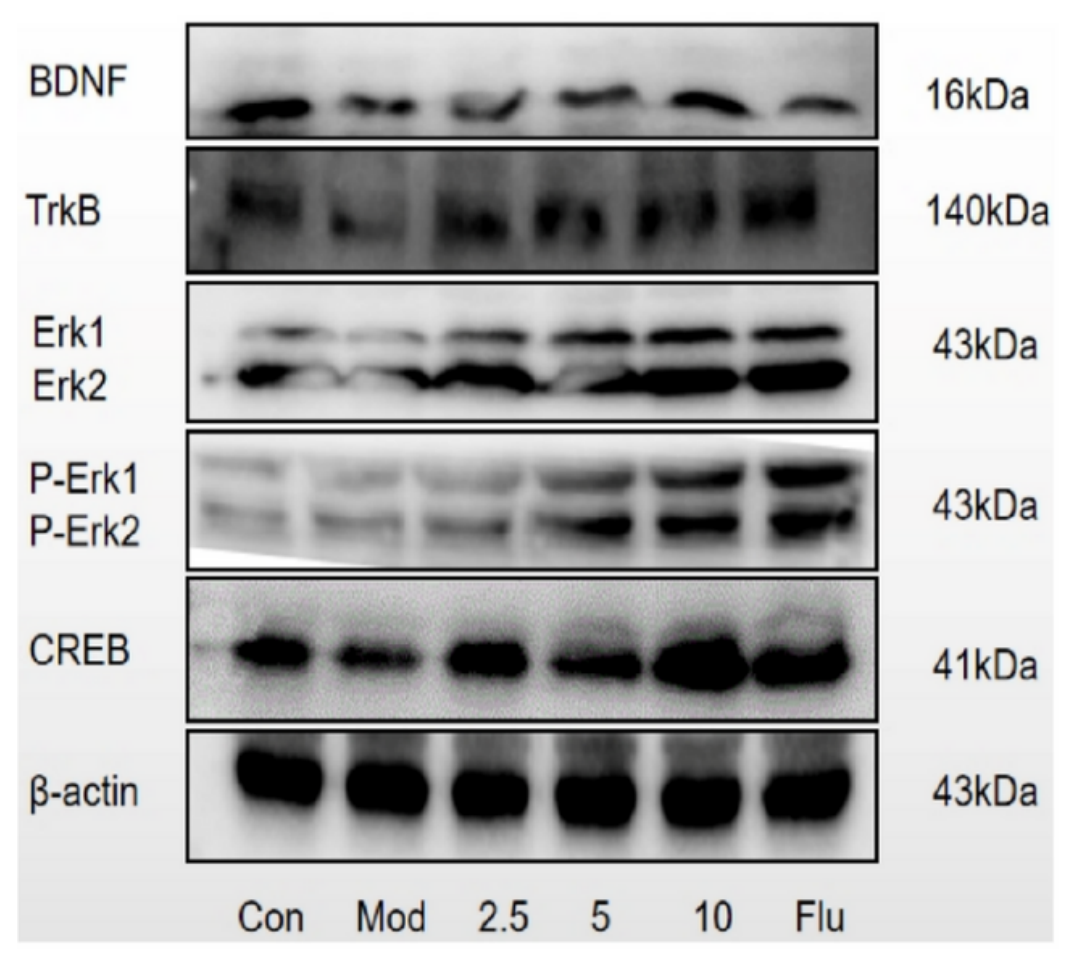

b

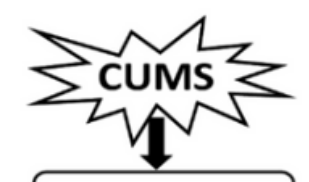

Serotonin

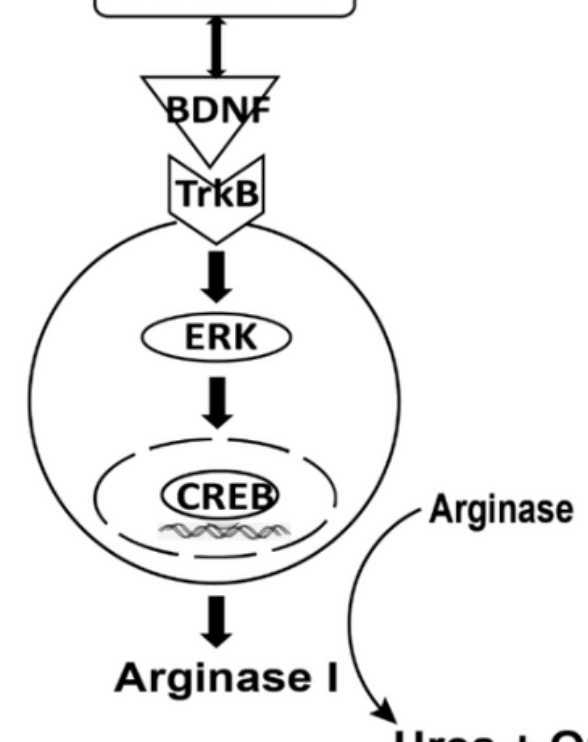

Urea + Ornithine

Figure 4

ELISA assays assessing serum Arg I and serotonin in different groups of rats. a: Arginase I, b: serotonin $(* \otimes P<0.05 ; * \star \otimes P<0.01 \rrbracket * \star \star \rrbracket P<0.001)$ 
a

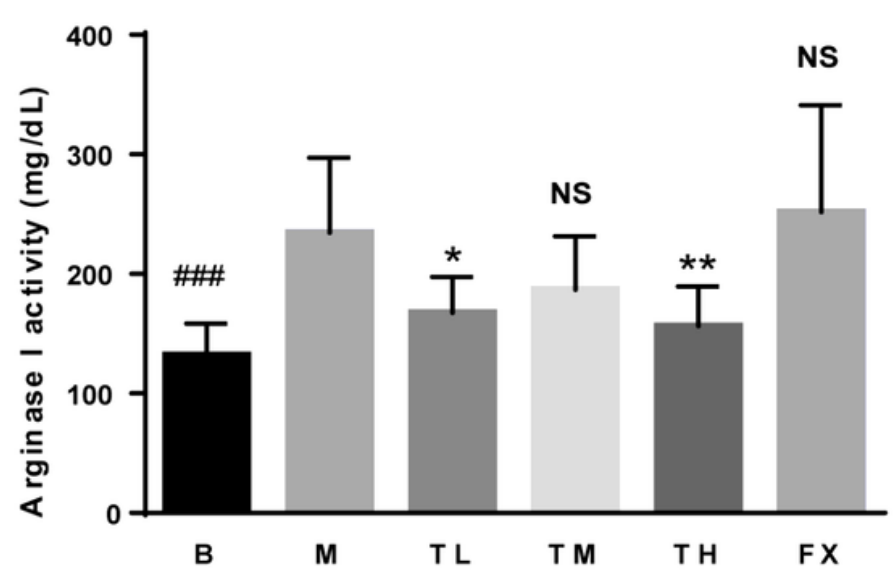

b

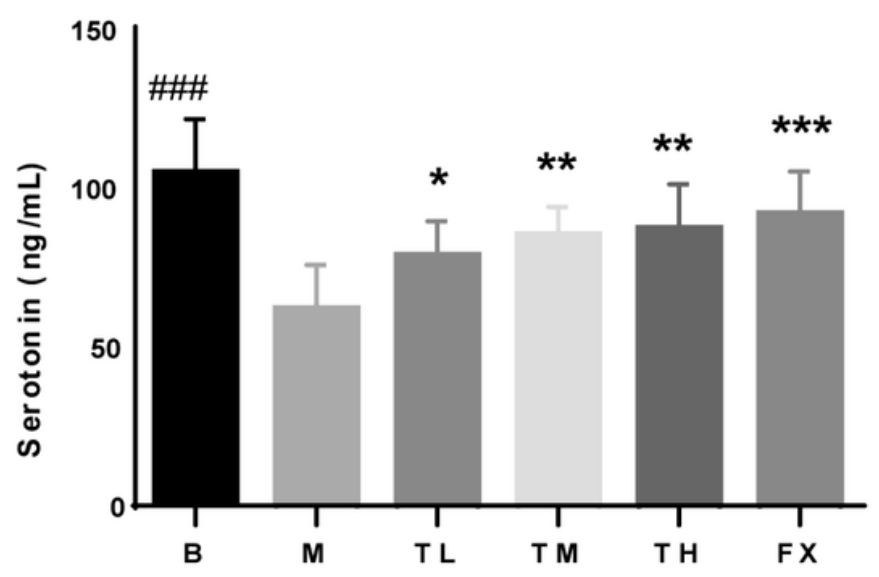

Figure 5

The effect of TSD on the expressions of upstream proteins of Arg I. a: targeted hippocampus protein expressions comprised in the BDNF/ERK/CREB signaling. b: Summary schema of determined varied metabolites and proteins involved in the BDNF-CREB-Arg I axis. Arg I is the enzyme that catalyzes the conversion from arginine to ornithine and urea. The transcription of $\mathrm{Arg} I$ is regulated by the expression of CREB, which is downstream of serotonin/BDNF/TrkB signaling. The BDNF-CREB-Arg I axis is downregulated after the induction of CUMS modeling and can be reversed by TSD administration.

\section{Supplementary Files}

This is a list of supplementary files associated with this preprint. Click to download.

- SupplementaryData.docx 